\title{
An overview of chiral separations of pharmaceutically active substances by HPLC (2018-2020)
}

\author{
Sofiya Grybinik ${ }^{1} \cdot$ Zuzana Bosakova $^{1}$ (i)
}

Received: 30 April 2021 / Accepted: 1 August 2021 / Published online: 24 August 2021

(c) Springer-Verlag GmbH Austria, part of Springer Nature 2021

\begin{abstract}
This review provides a brief survey of chiral separation of pharmaceutically active substances published over the last 3 years (2018-2020). Chiral separation of drugs is an important area of research. The control of enantiomeric purity and determination of individual enantiomeric drug molecules is a necessity especially for clinical, analytical, and regulatory purposes. Among chromatographic resolution methods, high-performance liquid chromatography based on chiral stationary phases remains the most popular and effective method used for chiral separation of various drugs. In this review, attention is paid to several classes of chiral stationary phases that have been the most frequently used for drug enantioseparation during this period.
\end{abstract}

\section{Graphic abstract}

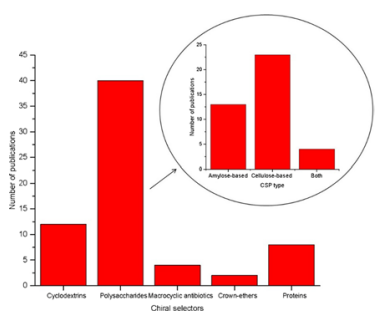

Keywords Chiral resolution $\cdot$ Pharmaceutical compounds $\cdot$ HPLC $\cdot$ Chiral stationary phase

\section{Introduction}

The term chirality is applicable for chemical substances that can have different spatial orientations of atoms or groups of atoms within one molecule. When multiple molecules only differ in their spatial orientations, they can be named stereoisomers and when two stereoisomers are mirror images of each other, they are called enantiomers. Enantiomers are optically active since they can exhibit clockwise or anticlockwise optical rotation of plane polarized light [1]. Many drugs already on the market as well as drugs awaiting marketing authorization are chiral and their enantioseparation or

Zuzana Bosakova

bosakova@natur.cuni.cz

1 Department of Analytical Chemistry, Faculty of Science, Charles University, Prague, Czech Republic determination of the optical purity is an indispensable step in the drug development process. Tragic experience with the administration of thalidomide racemate to pregnant women in the 1960s led to multiple congenital abnormalities in newborn babies. This case showed the ultimate importance of thorough analysis of any chiral compound intended for therapeutic use [2]. Nowadays, pharmaceutical companies must follow rigorous requirements defined by different regulatory authorities to ensure drug safety and prevent the recurrence of a similar incident. To ensure safe drug production, it is important to develop and optimize analytical and preparative techniques and approaches for the analysis and purification of enantiomers [3]. In addition, there is no doubt that chiral separations will gain even more interest and will be given even greater importance. For example, the international response to the Covid-19 pandemic has required the accelerated research of new drugs. During this research it was 
discussed that $S$-chloroquine and $S$-hydrochloroquine could potentially have a higher response against SARS-CoV-2 than their enantiomers thus putting to the fore the necessity of chiral separation [4].

In general, there are two approaches in the analysis of enantiomers. The first technique is called indirect and involves derivatization of chiral molecule with an optically pure derivatizing agent. This reaction results in the formation of diastereomers which can be separated in achiral environment. For the second approach, either a chiral mobile or stationary (CSP) phase is applied. For practical reasons, the CSP is used more often [3]. Today, it is possible to choose from a great variety of different commercially available CSPs. The development of CSPs is a constantly ongoing process. At a basic level, CSPs can be classified according to the structure of the chiral selector (CS) that makes up the main part of the stationary phase or, according to the predominant interaction mechanism provided by the CSP. The main types of CSs include cyclodextrin-, macrocyclic glycopeptide-, protein-, crown ethers- and polysaccharidebased CS [4].

The aim of this review is to provide an overview of the latest available publications that focus on the analysis of approved drugs and promising biologically active ingredients using different CSPs. The most common CSPs will be discussed in the context of their application. The latest approaches and methods of chiral drug analysis will also be included. Due to the accelerating development of new drugs, this review covers publications on chiral separations of pharmaceutical compounds over the last 3 years, from 2018 to early 2021. Most publications concern liquid chromatography, however, some studies also mention capillary electrophoresis. An overview of column names, type of bound CSs, separated analytes, and mobile phase compositions, discussed in this overview, is given in Table 1. A distribution of different types of CSs (CSPs in the inserted graph) used for the enantioseparation of drugs is shown in Fig. 1. To our best knowledge, no similar reviews have been published on this topic covering the time period in question.

\section{Cyclodextrin-based stationary phases}

Cyclodextrin-based CSPs have been shown to be widely applicable for the separation of enantiomers, diastereomers, structural isomers, and routine compounds [5]. Cyclodextrins (CDs) are a group of cyclic oligosaccharides which are produced through enzymatic reaction of glucosyl transferase on starch solutions. Although CDs comprising of up to 13 glucopyranose units exist, only three of them $-\alpha$-, $\beta$ - and $\gamma$ - with 6,7 , and 8 units, respectively, are of particular significance for analytical applications [6-8]. The different number of units in the structure of CDs results in their ability to form the host-guest complex with various compounds depending on their size and shape. The fact that $\beta$-cyclodextrin $(\beta-C D)$ exhibits stronger interactions with the majority of common drugs led to its wider use [9]. The trend over the last few years clearly shows that most of the CD-based stationary phases used to separate enantiomeric drugs are $\beta$-CD-based CSPs [10]. It is common to use derivatized $\beta-C D$ to enhance their enantioselectivity. A newly synthesized 4-chlorophenylcarbamate- $\beta$-CD CSP was evaluated for enhanced enantioseparation in HPLC. Several antifungal agents, proton pump inhibitors, and dihydropyridine calcium antagonists were analyzed and the CSP was shown to be stable providing good reproducibility for almost all the tested analytes [11]. 4-Chlorophenylcarbamate- $\beta$-CD CSP was also tested for separation and determination of chiral alminoprofen in rat plasma [12], as well as of flurbiprofen enantiomers [13]. Compared to Chiralpak OJ-RH (cellulose tris(4-methylbenzoate)) and Chiralpak AD-RH (amylose tris(4-methylbenzoate)) columns, this CSP provided better resolution and shorter analysis time under conditions compatible with mass spectrometric (MS) detection. A stilbene diamido-bridged bis( $\beta$-CD)-bound CSP was prepared demonstrating excellent $\beta$-blockers enantioseparation ability compared to a conventional $\beta$-CD-based CSP [14].

Enantioseparation of several non-steroidal anti-inflammatory drugs (ketoprofen, ketorolac, indoprofen, fenoprofen, suprofen, ibuprofen, and flurbiprofen) was performed using a dynamically coated pillar array column with hydroxypropyl$\beta$-CD as a CS with improved enantioresolution [15]. Several works published the use of $\beta$-CD derivatives in counter current chromatography. Successful preparative separation of 2-(4-bromomethylphenyl)propionic acid enantiomers, one of which is a precursor for further synthesis of chiral loxoprofen, was reported using hydroxypropyl- $\beta$-CD CS added to the aqueous phase by counter current chromatography [16]. An effective antiemetic drug ondansetron was also enantioseparated by counter current chromatography with sulfobutyl ether $\beta$-CD as a CS [17].

Capillary electrophoresis (CE) is another popular method for performing enantioseparation of pharmaceuticals with various $\beta$-CD-based CSs $[18,19]$. In CE, the combination of a CS with ionic liquids can help achieve better enantioselectivity [20]. Econazole and sulconazole, which were extracted from pharmaceutical creams for the first time, were successfully enantioseparated by capillary electrokinetic chromatography using a dual system containing hydroxypropyl$\beta-\mathrm{CD}$ and tetrabutylammonium L-lysine as the ionic liquid [21]. CE enantioseparation of antifungal ketoconazole and miconazole present in cream samples, based on their inclusion interactions with $\beta$-CD and hydroxypropyl- $\beta$-CD selectors, was also reported [22]. For the first time, environmentally friendly chiral separation was demonstrated using deep eutectic solvent choline chloride-D-sorbitol as an additive 


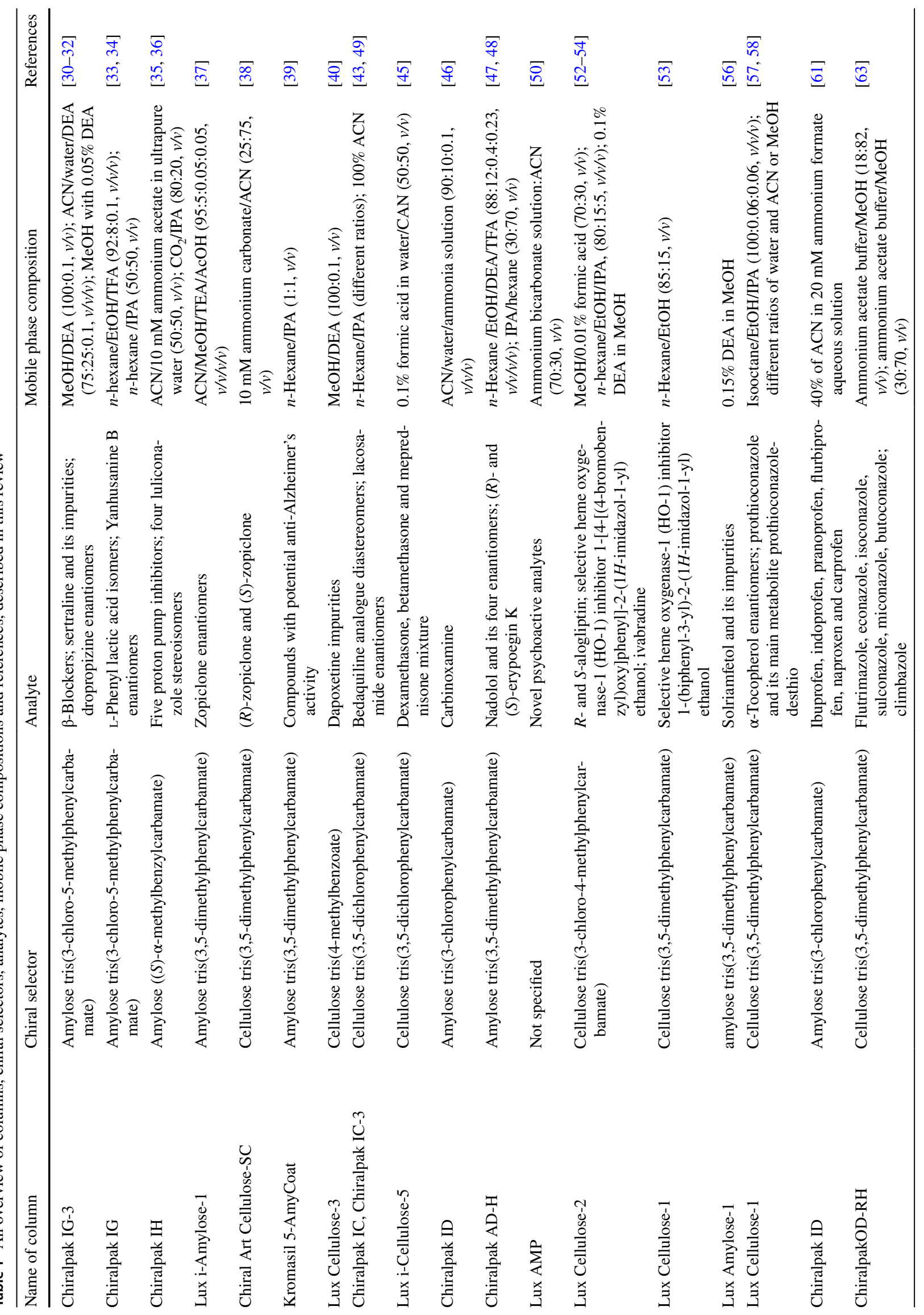




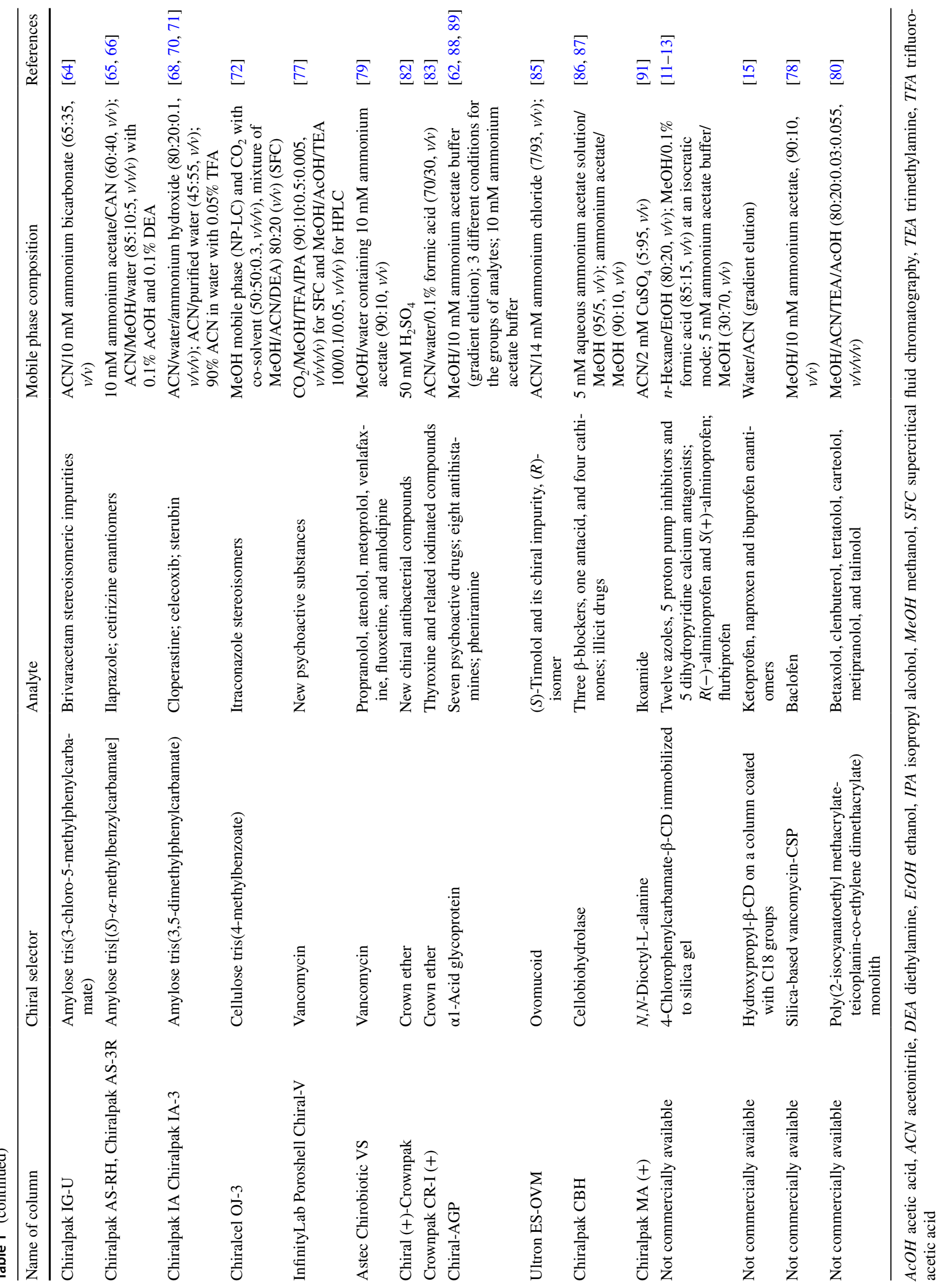


Fig. 1 Graph representing the distribution of different types of CS (CSPs in the inserted graph), used for enantioseparation of drugs described in this review

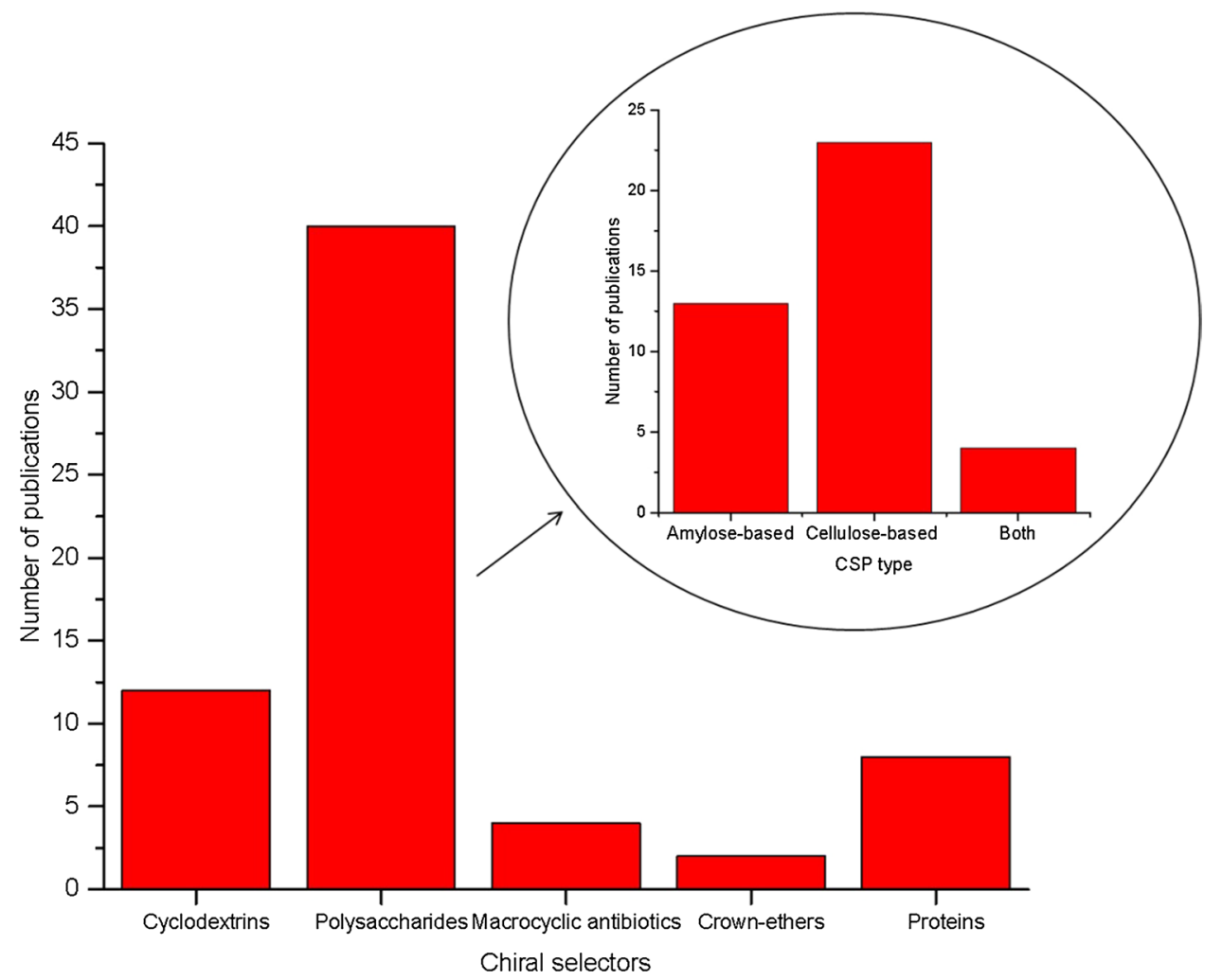

in succinyl- $\beta$-CD-electrokinetic chromatography for separation of lacosamide enantiomers. The deep eutectic solvent was reported as one of the greener alternatives to ionic liquids and the method proved to be suitable for the analysis of pharmaceutical formulations in compliance with regulatory requirements [23].

Enantioselective composite polyester membranes with bound derivatized hydroxypropyl- $\beta$-CD were prepared and provided better enantioselectivity for $R, S$-warfarin and $R, S$ nefopam compared to non-derivatized $\beta$-CD [24]. A newly introduced vortex-assisted matrix solid-phase dispersion method was published using $\beta-C D$ as a CS to extract the ibuprofen enantiomers from the breast milk samples with good recovery [25].

\section{Polysaccharide-based stationary phases}

Polysaccharide-based stationary phases have been pioneered by Okamoto et al. [26-28]. They are prepared on the base of cellulose or amylose two optically active biopolymers. It was found that derivatized polymers exhibited much better enantioselectivity which led to the development of different modified stationary phases. Previously, the columns had to be designed separately for reverse-phase and for normal-phase separation mode (coated). In recent developments, one (immobilized) column can operate in normal-, reverse-phase, and polar-organic modes as well as in HILIC mode and several types of columns are also available with a smaller particle size $(3 \mu \mathrm{m})$. It provides new application possibilities [29]. Many new articles on chiral drug separation mention the use of polysaccharide-based CSPs. A schematic representation of Chiralpak columns based on amylose and cellulose derivatives immobilized on silica gel is shown in Fig. 2. A Chiralpak IG-3 column based on immobilized amylose tris(3-chloro-5-methylphenylcarbamate), particle size $3 \mu \mathrm{m}$, seems to be a popular choice for separation of different pharmaceuticals. It was used for separation of $\beta$-blockers under polar-organic, reversed-phase and HILIC separation modes. Separation efficiency was shown to be higher under HILIC conditions, which is potentially useful for shortening analytical times [30]. A successful single-run separation in the reversed-phase separation mode allowed the determination of chiral purity of sertraline and direct quantification of dropropizine enantiomers in rat plasma. Both separations were achieved on a Chiralpak IG-3 column [31, 32]. The same column was used for enantioseparation of some naturally occurring compounds with therapeutic properties, e.g., a novel antimicrobiologic agent L-phenyl lactic acid purified from Lactobacillus plantarum ZJ316 [33]. Isoquinoline-derived alkaloid enantiomers from Corydalis yanhusuo plant, which possess analgesic, antihypertensive, antimalarial, antitumor, and sedative properties, were also resolved on a Chiralpak IG column [34]. Simultaneous enantiomeric analysis of five proton pump inhibitors extracted from soil and sediment samples 


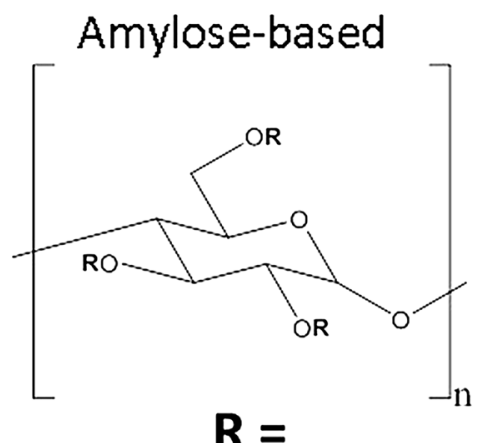

\section{ChiralpakIA}

Amylose tris(3,5-dimethylphenylcarbamate)<smiles>CC(=O)Nc1cc(C)cc(C)c1</smiles>

Chiralpak IG

Amylose tris(3-chloro-5-methylphenylcarbamate)<smiles>CC(=O)Nc1cc(C)cc(Cl)c1</smiles>

\section{ChiralpakID}

Amylose tris(3-chlorophenylcarbamate)

Amylose tris(3-chloro-4-methylphenylcarbamate)<smiles>CC(=O)Nc1cccc(Cl)c1</smiles><smiles>CC(=O)Nc1ccc(C)c(Cl)c1</smiles>

ChiralpakIH

Amylose tris[(S)- $\alpha$-methylbenzylcarbamate]<smiles>CC(=O)N[C@@H](C)c1ccccc1</smiles>

\section{Cellulose-based}

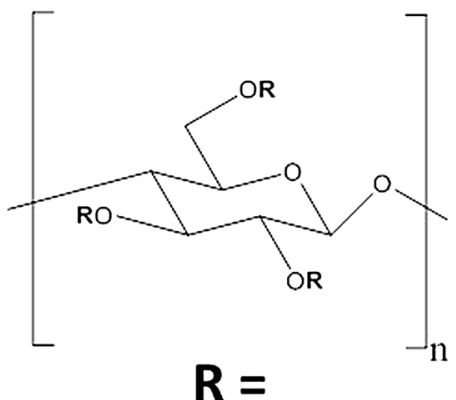

\section{Chiralpak IB}

Cellulose tris(3,5-dimethylphenylcarbamate)<smiles>CC(=O)Nc1cc(C)cc(C)c1</smiles>

\section{ChiralpakIC}

Cellulose tris(3,5-dichlorophenylcarbamate)<smiles>CC(=O)Nc1cc(Cl)cc(Cl)c1</smiles>

Fig. 2 Schematic representation of Chiralpak columns based on amylose and cellulose derivatives immobilized on silica gel 
using QuEChERS (Quick, Easy, Cheap, Effective, Rugged, Safe) technique was performed on an immobilized amylose tris $((S)$ - $\alpha$-methylbenzylcarbamate)-based Chiralpak IH column [35]. The same column was selected for the separation of four stereoisomers of the antifungal agent luliconazole by supercritical fluid chromatography (SFC) [36]. Five different columns based on both derivatized cyclofructans (Larihc CF6-RN and Larihc CF6-P columns) and teicoplanin glycopeptide (Chirobiotic T column) were tested for enantioseparation of zopiclone, a drug used to treat insomnia. None of them showed sufficient enantioresolution. Finally, the Lux i-Amylose- 1 column (immobilized amylose tris(3,5-dimethylphenylcarbamate)) provided satisfactory results enabling validation of the method and its further use for the analysis of zopiclone in tablets [37]. The $(R)$ - and $(S)$-zopiclone were also determined in human hair on a Chiral Art Cellulose-SC column based on immobilized cellulose tris(3,5-dichlorophenylcarbamate) using previous optimized extraction to prevent decomposition of the analytes [38]. A Kromasil 5-AmyCoat column, containing amylose tris(3,5-dimethylphenylcarbamate) CS, was used to separate compounds with potential pharmacological activity against Alzheimer's disease during their development [39].

Many articles deal with the screening of polysaccharidebased columns to select the most suitable column for a given purpose, i.e., the analysis of the compound(s) of interest. Simultaneous determination of achiral and chiral impurities of a commercially available dapoxetine, prescribed for the treatment of premature ejaculation in men, and a counterfeit drug ordered on the internet, was conducted on seven polysaccharide-based columns and the Lux Cellulose-3 (cellulose tris(4-methylbenzoate)) column proved to be the best [40]. Four different cellulose- and amylose-based columns were tested to investigate the retention behavior of several antiretroviral drugs. The retention mechanism was shown to depend on the chemical structure of the compounds and no universal column could be found for successful enantioseparation of all analytes [41]. It was found that lamivudine, efavirenz, and darunavir were retained more on cellulosebased stationary phases due to the increased involvement of hydrogen bonds. On the other hand, abacavir, dolutegravir, tenofovir, and emtricitabine were better resolved on amylose-based stationary phases in which the dominant retention mechanism is associated with the presence of well-defined cavities involved in steric interactions with analytes [41]. In addition to the separation of econazole by electrokinetic chromatography with hydroxypropyl- $\beta-\mathrm{CD}$ and tetrabutylammonium L-lysine [21], another new study focused on the separation of econazole enantiomers extracted from rat plasma by HPLC with tandem mass spectrometric detection (HPLC-MS/MS) [42]. Three polysaccharide-based CSPs were tested, namely Chiralpak IA (immobilized amylose tris(3,5-dimethylphenylcarbamate)), Chiralpak IC (immobilized cellulose tris(3,5-dichlorophenylcarbamate)), and Chiralpak ID (immobilized amylose tris(3-chlorophenylcarbamate)) columns and Chiralpak IC was selected as the most suitable. The recovery, precision and the analysis time were similar for both methods, but limit of detection and limit of quantitation as well as linearity range were much better with the HPLC-MS/MS method [21, 42]. A Chiralpak IC column was also used for the separation of eight bedaquiline analogue diastereomers [43]. The retention behavior of several local anesthetics was investigated on five different cellulose-based columns, namely: Lux Cellulose-1 (cellulose tris(3,5-dimethylphenylcarbamate)), Lux Cellulose-2 (cellulose tris(3-chloro-4-methylphenylcarbamate)), Lux Cellulose-3, Lux Cellulose-4 (cellulose tris(4-chloro3-methylphenylcarbamate)), Lux i-Cellulose-5 (immobilized cellulose tris(3,5-dichlorophenylcarbamate)), and on three amylose-based columns, namely Lux Amylose-1 (amylose tris(3,5-dimethylphenylcarbamate)), Lux Amylose-2 (amylose tris(5-chloro-2-methylphenylcarbamate)), and Lux i-Amylose-3 (immobilized amylose tris(3-chloro-5-methylphenylcarbamate)). Of all five cellulose columns, the Lux i-Cellulose- 5 showed the highest retention values for all tested compounds. This was attributed to the presence of two chlorine atoms in the structure of this CSP, probably responsible for chiral discrimination. For amylose columns, their enantioseparation ability was generally low [44]. Of the several different CSPs based on amylose and cellulose, Lux i-Cellulose- 5 column in reverse-phase separation mode was the most suitable for the enantioseparation of synthetic corticosteroids. The method was used for confirmed identification and quantitation of meprednisone, a prohibited substance in horse racing [45]. Chiral separation and determination of enantiomers of antihistamine drugs (meclizine, cloperastine, azelastine, carbinoxamine and mequitazine) in rat plasma were performed on both Chiralpak IA and Chiralpak ID columns to achieve a good enantioresolution especially towards achieving the baseline separation of carbinoxamine [46]. Determination of nadolol and its four enantiomers in human plasma was conducted on a Chiralpak AD-H column (coated amylose tris(3,5-dimethylphenylcarbamate)) [47]. The same column was used for the enantioseparation of $(S)$ - and $(R)$-erypoegin $\mathrm{K}$ to examine their apoptosis-inducing and anti-proliferative activities in HL-60 cells. It was found out that only S-isomer exhibits this activity [48]. Determination of $R$-lacosamide in human plasma was performed on a Chiralpak IC-3 column (particle size $3 \mu \mathrm{m}$ ), where its isomers were completely resolved [49]. The Lux AMP column, primarily intended for the analysis of synthetic chiral drugs such as amphetamine and 3,4-(methylenedioxy)methamphetamine, was used for enantioseparation of new psychoactive drugs, and 88 of 95 drugs tested were successfully enantioseparated at $\mathrm{pH} 11.3$ without stationary phase damage [50]. A Lux Cellulose-2 was 
applied for the analysis of alogliptin, a selective inhibitor of the enzyme dipeptidyl peptidase-4 (used to treat diabetes) [51, 52]. Both this column and Lux Cellulose-1 column were shown to be useful for enantioseparation of selective heme oxygenase-1 inhibitors [53]. Ivabradine is a heart-rate-lowering agent and only $S$-isomer is prescribed for the treatment of heart failure. Seven different Lux amylose- and cellulosebased columns were tested to resolve ivabradine enantiomers and only Lux Cellulose- 2 was successful. The method was validated and used to analyze $R$-enantiomer content below $0.05 \%$ in tablet $[54,55]$. A similar study was performed for a new potential drug to treat patients suffering with narcolepsy. As in the previous case, the same seven polysaccharide-based columns were screened to obtain separation of solriamfetol chiral impurities and in this case the Lux Amylose-1 column was selected for the method validation [56]. A method for the separation of $\alpha$-tocopherol enantiomers was successfully developed with a Lux Cellulose-1 column [57]. Several polysaccharide-based CSPs were tested for enantiomeric separation of the antifungal agent prothioconazole and its metabolite prothioconazole-desthio, and the Lux Cellulose-1 column was rated as the best [58]. A comparative study was performed with chiral basic drugs on amylose- and cellulose phenylcarbamate-based chiral columns with a focus on substituent electron-donor and electronacceptor effects. In general, amylose-based CSPs were found to provide better enantioselectivity [59]. Similar enantioselective behavior on several Chiralpak amylose- and cellulose-based columns with immobilized stationary phases was observed for $\beta$-carboline analogues, which have great pharmacological potential due to their antimicrobial and antiHIV effects, and for tetrahydroisoquinoline and benzazepine analogues of pharmaceutical importance [60]. Another study focused on enantioseparation of seven non-steroidal antiinflammatory profens and etodolac and naproxen enantiomers in fish tissues, performed on four cellulose- and amylosebased columns showed that a Chiralpak ID column again provided the best resolution of all profens. However, enantiomers of etodolac could only be resolved on cellulosebased CSP [61]. On the other hand, neither Chiralpak ID, nor Chiralcel OJ-RH (coated cellulose tris(4-methylbenzoate)) columns provided sufficient resolution of antipsychotic drug enantiomers in a study to analyze these compounds related to their use for sedative breeding purposes. Only an alpha-1-acid glycoprotein Chiralpak AGP column in combination with a mobile phase containing only methanol as an organic modifier made it possible to develop a suitable method for the enantioseparation of all analytes [62]. The Chiralcel OD-RH (coated cellulose tris(3,5-dimethylphenylcarbamate)) column usable in reverse-phase separation mode provided full resolution of four of seven imidazoles and partial resolution of the remaining three antifungal drugs under optimized conditions [63]. A Chiralpak IG column was used to analyze stereoisomeric impurities of brivaracetam [64]. Separation and quantification of ilaprazole enantiomers in rat plasma was performed on a Chiralpak AS-RH (coated amylose tris $((S)-\alpha$-methylbenzylcarbamate $)$ ) column allowing MS detection with proper mobile phase compositions [65]. A Chiralpak AS-3R column (particle size $3 \mu \mathrm{m}$ ) was used for separation of $( \pm)$-cetirizine and enantiopurity assessment of $R$-levocetirizine [66]. Separation of 12 antifungal agent posaconazole related stereoisomers by multiple heart-cutting chiral-chiral two-dimensional LC method was performed on a Chiralpak IB column (immobilized cellulose tris(3,5-dimethylphenylcarbamate) in 1D mode and on Chiralpak IF3 (immobilized amylose tris(3-chloro-4-methylphenylcarbamate)) and Chiralpak IC columns in 2D mode. The 2D mode was chosen as better because complete separation in 1D mode was not achieved for all stereoisomers [67]. A comparative study was performed on four Chiralpak (IA, IB, IC, and ID) columns and one protein-based Chiralpak AGP column to separate enantiomers of cloperastine, an antitussive agent. Chiralpak IA column showed the best resolution and peak shapes along with a shorter retention time [68]. This column was the only one which was able to separate all new chiral azoles in another comparative study with several polysaccharide-based columns with various CSs [69]. The same column demonstrated good enantioselectivity in the analysis of seven process-related impurities of celecoxib as well as for enantiomers of sterubin, a potential neuroprotector extracted from Eriodictyon californicum plant [70, 71]. An interesting comparative study was performed on a Chiralcel OJ-3 (particle size $3 \mu \mathrm{m}$ ) column using both SFC and NP-LC. Both methods provided satisfactory results, however, SFC is considered to be a more ecological method requiring lower solvent consumption [72].

\section{Macrocyclic antibiotics-based stationary phases}

Macrocyclic antibiotics were first introduced as CSPs by Armstrong et al. in 1994 [73]. They represent chemical compounds possessing various functional groups that can participate in chiral recognition (hydroxyl, amine, and carboxyl groups, aromatic moieties etc.). Compared to other available CSPs, the macrocyclic antibiotics are complementary to each other, have particularly good stability and capacity and they can operate in all three separation modes (normal, reverse-phase, and polar-organic). The most common antibiotics used for the preparation of these stationary phases include vancomycin, teicoplanin, rifamycin and their analogues [74-76].

In the recent years, prevalence for the use of vancomycin-based CSPs can be observed (for vancomycin CS, see Fig. 3). Successful enantioseparations of 40 


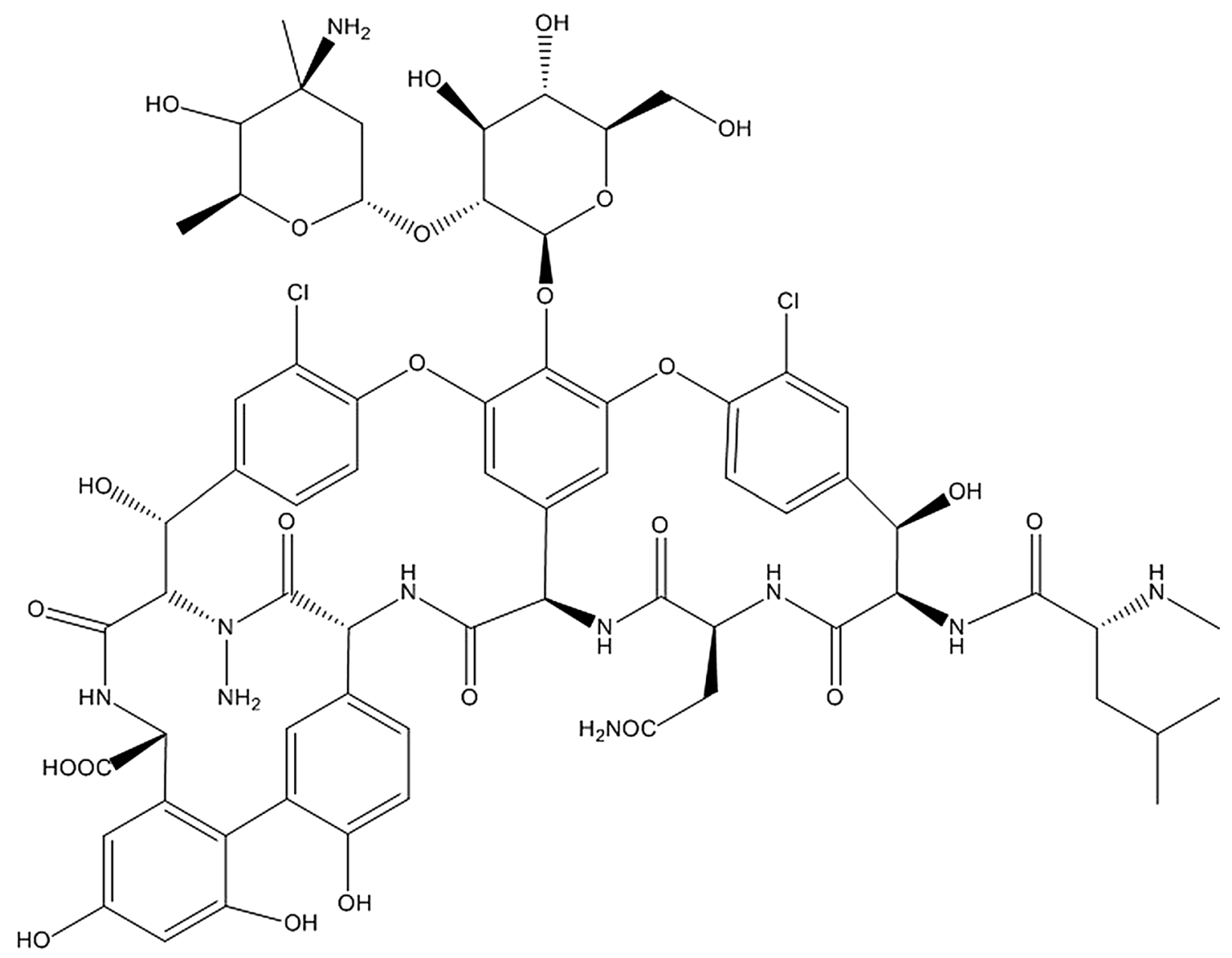

Fig. 3 Structure of vancomycin CS

new psychoactive drugs were obtained on superficially porous particle vancomycin-based CSPs in HPLC and SFC. Base-line separation of individual substances was achieved in either one or the another separation system, therefore, the techniques were regarded as complementary [77]. An interesting study focused on the analysis of baclofen enantiomers in human plasma using vancomycin-based silica stationary phase by nano-liquid chromatography [78]. Vancomycin-bonded stationary phase was applied for enantioseparation of propranolol, atenolol, metoprolol, venlafaxine, fluoxetine, and amlodipine and the mechanism of chiral recognition was evaluated [79].

However, the use of other macrocyclic antibiotics was also published. For the first time, successful preparation of teicoplanin functionalized polymeric monolith based on poly(2-isocyanatoethyl methacrylate-teicoplanin-co-ethylene dimethacrylate) and its application for the enantioseparation of betaxolol, clenbuterol, tertatolol, carteolol, metipranolol, and talinolol was published. This newly prepared CSP provided good reproducibility and enantioselectivity [80].

\section{Crown ether-based stationary phases}

Chiral crown ethers are known to be especially useful for HPLC and CE enantioseparations of primary amine compounds although separations of secondary amines or even amides have been reported [81]. There are a limited number of publications mentioning the usefulness of crown ether-based stationary phases for the enantioseparation of pharmacologically active compounds. A chiral Crownpak CR (+) column was used for chiral resolution of four stereoisomers of newly synthesized antibacterial agents with two chiral centers. In this study, the importance of hydrogen bonds in the process of chiral recognition was emphasized [82]. Chiral thyroxine impurities were successfully characterized and subsequently determined in pharmaceutical tablets on the Crownpak CR-I (+) column based on chiral crown ether immobilized to silica gel [83]. 


\section{Protein-based stationary phases}

Protein-based CSPs are another class of stationary phase that allow enantioseparation of various chiral compounds. However, the introduction of macrocyclic antibiotics reduced their use mainly due to significantly reduced flexibility and the limitation of the content of organic modifier in the mobile phase. However, currently available protein-based columns are stable enough to operate in all three separation modes. The most commonly used protein-based CSPs include alpha-1-acid glycoprotein, bovine serum albumin and cellobiohydrolases CSs [84]. The enantiomeric purity of timol maleate was performed on the Ultron ES-OVM column based on an ovomucoid CS. The ecologically acceptable method was validated and used for the analysis of $(S)$-timolol and its chiral impurity $(R)$-isomer in eye drops [85]. A comparison of Lux Cellulose-1 and Chiralpak CBH (based on protein cellobiohydrolase) columns in polar-organic and reversephase modes was performed for the analysis of several $\beta$-blockers and omeprazole. The results obtained showed a better overall efficiency of the Chiralpak CBH column [86]. A Chiralpak CBH column was also selected for the analysis of illicit drugs in wastewater and surface water to develop a method for daily monitoring of these compounds [87]. Enantioselective separation of eight antihistamines and chiral separation of pheniramine and its enantioselective pharmacokinetics in rat plasma were performed on an alpha-1-acid glycoprotein-based Chiralpak AGP column $[88,89]$. The use of a Chiralpak AGP column for the analysis of $S$-(+)-escitalopram, a selective serotonin reuptake inhibitor, was also described [90]. A Daicel Chiralpak MA $(+)$ stationary phase composed of $N, N$-dioctyl-L-alanine was used for determination of the absolute configuration of ikoamide, an antimalarial lipopeptide from Okeania sp. marine cyanobacterium [91].

\section{Conclusion}

This review summarizes the scientific literature relating to the HPLC analysis of chiral pharmaceutical compounds from 2018 to 2020. It is obvious that polysaccharide-based CSPs are the most popular choice followed by CD-based stationary phases and those based on macrocyclic antibiotics. Several articles mention the use of crown etherand protein-based CSPs, as well as the involvement of CE with CD-based CSs, but they have limited application. The popularity of polysaccharide-based CSPs is growing due to the introduction of CSPs based on newly synthesized polysaccharide derivatives, the development of techniques for more efficient covalent immobilization of these chiral derivatives onto silica, and the introduction of superficially porous silica as a carrier. This, together with the smaller particle size, leads to an extended range of applications and higher separation efficiency in shorter analysis times.

Acknowledgements This research was funded by the Grant Agency of Charles University, SVV260560.

\section{References}

1. Nag A (2018) Asymmetric synthesis of drugs and natural products. CRC Press, Boca Raton

2. McBride WG (1961) Lancet 278:1358

3. Witte DT (1993) Pharm World Sci 15:283

4. Armstrong DW, Zhang B (2001) Anal Chem 73:557A

5. Ward TJ, Armstrong DW (1986) J Liq Chromatogr 9:407

6. Tiwari G, Tiwari R, Rai AK (2010) J Pharm Bioallied Sci 2:72

7. Szejtli J (1998) Chem Rev 98:1743

8. Scriba GKE (2019) Trends Anal Chem 120:115639

9. Wren S (2001) The separation of enantiomers by capillary electrophoresis. Vieweg+Teubner Verlag, Wiesbaden

10. Zhang Y, Guo Z, Ye J, Xu Q, Liang X, Lei A (2008) J Chromatogr A 1191:188

11. Sun J, Ma S, Liu B, Yu J, Guo X (2019) Talanta 204:817

12. Sun J, Zhu Y, Lin B, Yu J (2020) J Pharm Biomed Anal 191:113552

13. Sun J, Liu B, Cai L, Yu J, Guo X (2020) New J Chem 44:10334

14. Shuang Y, Zhang T, Li L (2020) J Chromatogr A 1614:460702

15. Naghdi E, Fakhari AR, Baca M, De Malsche W (2020) J Chromatogr A 1615:460752

16. Zhang H, Qiu X, Lv L, Sun W, Wang C, Yan J, Tong S (2018) J Sep Sci 41:2828

17. Sun W, Bao H, Qian J, Tong S (2020) J Sep Sci 44:922

18. Casado N, Valimaña-Traverso J, García MÁ, Marina ML (2020) Crit Rev Anal Chem 50:554

19. Solínová V, Kasicka V, Sázelová P, Holý A (2009) Electrophoresis 30:2245

20. Řezanka P, Navrátilová K, Řezanka M, Král V, Sýkora D (2014) Electrophoresis 35:2701

21. Salido-Fortuna S, Marina ML, Castro-Puyana M (2020) J Chromatogr A 1621:461085

22. Azhari NR, Yahaya N, Mohd Suah FBM, Prabu S, Hui BY, Shahriman MS, Mohamad Zain NNM, Raoov M (2021) Chirality 33:37

23. Salido-Fortuna S, Casado N, Castro-Puyana M, Marina ML (2021) Microchem J 160:105669

24. Ke J, Yang K, Bai X, Luo H, Ji Y, Chen J (2021) Sep Purif Technol 255:117717

25. León-González ME, Rosales-Conrado N (2017) J Chromatogr A 1514:88

26. Yashima E, Okamoto Y (1995) Bull Chem Soc Jpn 68:3289

27. Yashima E, Yamamoto C, Okamoto Y (1998) Synlett 1998:344

28. Yashima E (2001) J Chromatogr A 906:105

29. Cirilli R, Carradori S, Casulli A, Pierini M (2018) J Sep Sci 41:4014

30. Merino MED, Lancioni C, Padró JM, Castells CB (2020) J Chromatogr A 1634:461685

31. Rosetti A, Ferretti R, Zanitti L, Casulli A, Villani C, Cirilli R (2020) J Pharm Anal 10:610

32. Balaji N, Gabani BB, Todmal U, Sulochana SP, Saini NK, Chandran R, Mullangi R (2019) Biomed Chromatogr 33:e4434 
33. Zhou Q, Gu R, Li P, Lu Y, Chen L, Gu Q (2020) Appl Microbiol Biotechnol 104:5283

34. Wang LY, Qiu BL, Xia H, Xia GY, Xiao BB, Zhang JF, Zhong WC, Lin S (2020) J Nat Prod 83:489

35. Lun J, Ma S, Xue M, Zhao P, Song Y, Guo X (2021) Microchem J 160:105625

36. Pandya PA, Shah PA, Shrivastav PS (2020) J Chromatogr A 1625:461299

37. Kozlov O, Záhoráková D, Gondová T (2020) J Pharm Biomed Anal 190:113549

38. Miyaguchi H, Kuwayama K (2017) J Chromatogr A 1519:55

39. Catto M, Pisani L, de la Mora E, Belviso BD, Mangiatordi GF, Pinto A, Palma AD, Denora N, Caliandro R, Colletier JP, Silman I, Nicolotti O, Altomare CD (2020) ACS Med Chem Lett 11:869

40. Tóth G, Fogarasi E, Bartalis-Fábián G, Foroughbakhshfasaei M, Boldizsár I, Darcsi A, Lohner S, Scriba GK, Szabó ZI (2020) J Chromatogr A 1626:461388

41. Choppari T, Gunnam S, Chennuru LN, Boddala ChSR, Murthy CP, Talluri KMVN (2021) Chromatographia 84:297

42. Zhang J, Liu B, Jiang Z, Cui Y, Guo X (2020) Anal Biochem 602:113791

43. Jiang S, Wu C, Yu J, Sun T, Guo X (2019) Chirality 31:72

44. Pérez-Baeza M, Escuder-Gilabert L, Martín-Biosca Y, Sagrado S, Medina-Hernández MJ (2020) J Chromatogr A 1625:461334

45. Karatt KT, Sathiq MA, Laya S (2020) Steroids 155:108572

46. Li M, Zhang J, Ma S, Jiang Z, Di X, Guo X (2020) New J Chem 44:5819

47. Lee SB, Pham TV, Mai XL, Le TAT, Nguyen TNV, Kang JS, Mar W, Kim KH (2020) Anal Sci Technol 33:59

48. Hikita K, Saigusa S, Takeuchi Y, Matsuyama H, Nagai R, Kato K, Murata T, Tanaka H, Wagh YS, Asao N, Kaneda N (2020) Bioorg Med Chem 28:115490

49. Jalakam SP, Tambe VS, Deodhar MN, Prakya V, Waghmode J, Pawar P (2020) Pharm Chem J 54:96

50. Hägele JS, Basrak M, Schmid MG (2020) J Pharm Biomed Anal 179:112967

51. Andukuri R, Drincic A, Rendell M (2009) Diabetes Metab Syndr Obes 2:117

52. Kant R, Bodla RB, Bhutani R, Kapoor G (2019) Adv Pharm Bull 9:147

53. Ianni F, Carotti A, Intagliata S, Macchiarulo A, Chankvetadze B, Pittalà V, Sardella R (2020) Chromatographia 83:1509

54. Ferencz E, Kovács B, Boda F, Foroughbakhshfasaei M, Kelemen VK, Tóth G, Szabó ZI (2020) J Pharm Biomed Anal 177:112851

55. Tse S, Mazzola N (2015) Pharm Ther 40:810

56. Köteles I, Foroughbakhshfasaei M, Dobó M, Ádám M, Boldizsár I, Szabó ZI, Tóth G (2020) Chromatographia 83:909

57. Gill BD, Indyk HE (2020) J AOAC Int 103:12882

58. Liu H, Ding W (2019) Chirality 31:219

59. Matarashvili I, Chelidze A, Dolidze G, Kobidze G, Zaqashvili N, Dadianidze A, Bacskay I, Felinger A, Farkas T, Chankvetadze B (2020) J Chromatogr A 1624:461218

60. Ilisz I, Bajtai A, Szatmári I, Fülöp F, Lindner W, Péter A (2020) J Chromatogr A 1615:460771

61. Li M, Liang X, Guo X, Di X, Jiang Z (2020) Microchem J 153:104511

62. Zhu B, Li S, Zhou L, Li Q, Guo X (2019) Food Chem 300:125241

63. Liu Y, Cai L, Lun J, Zhao M, Guo X (2020) New J Chem 44:18337

64. Baksam V, Pocha SNVR, Chakka VB, Ummadi RR, Kumar P (2020) Chirality 32:1208
65. Ou F, Zhou Y, Lei J, Zeng S, Wu F, Zhang N, Yu L (2020) J Pharm Anal 10:617

66. Kannappan V, Kanthiah S (2020) J Anal Chem 75:349

67. Xu F, Xu Y, Liu G, Zhang M, Qiang S, Kang J (2020) J Chromatogr A 1618:460845

68. Lun J, Zhao P, Jiang Z, Song Y, Guo X (2020) Chirality 32:1129

69. Kenari ME, Putman JI, Singh RP, Fulton BB, Phan H, Haimour RK, Tse K, Berthod A, Lovely CJ, Armstrong DW (2021) Molecules 26:213

70. Tome T, Časar Z, Obreza A (2020) Molecules 25:809

71. Hofmann J, Fayez S, Scheiner M, Hoffmann M, Oerter S, AppeltMenzel A, Maher P, Maurice T, Bringmann G, Decker M (2020) Chem Eur J 26:7299

72. Agrawal R, Belemkar S, Bonde C (2020) Microchem J $158: 105320$

73. Armstrong DW, Tang Y, Chen S, Zhou Y, Bagwill C, Chen JR (1994) Anal Chem 66:1473

74. Xiao TL, Armstrong DW (2004) Enantiomeric separations by HPLC using macrocyclic glycopeptide-based chiral stationary phases. In: Gübitz G, Schmid MG (eds) Chiral separations. Methods in molecular biology, vol 243. Humana Press, Totowa

75. Aboul-Enein HY, Ali I (2000) Chromatographia 52:679

76. Honetschlagerova-Vadinska M, Srkalova S, Bosakova Z, Coufal P, Tesarova E (2009) J Sep Sci 32:1704

77. Folprechtová D, Kalíková K, Kadkhodaei K, Reiterer C, Armstrong DW, Tesařová E, Schmid MG (2021) J Chromatogr A 1637:461846

78. D'Orazio G, Fanali C, Gentili A, Tagliaro F, Fanali S (2019) J Chromatogr A 1605:360358

79. Li J, Liu R, Wang L, Liu X, Gao H (2019) Chirality 31:236

80. Luo R, Han H, Liu J, Xu D, Wang Q, Fanali S, Jiang Z (2020) J Pharm Biomed Anal 182:113129

81. Paik MJ, Kang JS, Huang BS, Carey JR, Lee W (2013) J Chromatogr A 1274:1

82. Ali I, Suhail M, Al-Othman ZA, Al-Mohaimeed AM, Alwarthan A (2020) Sep Purif Technol 236:116256

83. Lee Y, Bang E, Lee W, Na YC (2021) J Pharm Biomed Anal 196:113904

84. Bi C, Zheng X, Azaria S, Beeram S, Li Z, Hage D (2016) Separations 3:27

85. Mitrović M, Protić A, Malenović A, Otašević B, Zečević M (2020) J Pharm Biomed Anal 180:113034

86. Fu Y, Borrull F, Fontanals N, Marcé RM (2020) Chirality 32:876

87. Wang W, Guo C, Chen L, Qiu Z, Yin X, Xu J (2021) Environ Pollut 273:116424

88. Zhu B, Xue M, Liu B, Li Q, Guo X (2019) J Pharm Biomed Anal $176: 112803$

89. Liu Y, Zhu B, Xue M, Jiang Z, Guo X (2020) Microchem J 156:104989

90. Soliman SM (2019) Chirality 31:185

91. Iwasaki K, Iwasaki A, Sumimoto S, Matsubara T, Sato T, Nozaki T, Saito-Nakano Y, Suenaga KL (2020) J Nat Prod 83:481

Publisher's Note Springer Nature remains neutral with regard to jurisdictional claims in published maps and institutional affiliations. 